\title{
ENSENAAR BIOÉTICA: CÓMO TRASMITIR CONOCIMIENTOS, ACTITUDES Y VALORES
}

\author{
Francisco Javier León Correa*
}

\begin{abstract}
Resumen: En este trabajo se presentan experiencias docentes y reflexiones sobre cómo realizar la enseñanza de la bioética de modo que cumpla con tres objetivos y a la vez retos para el docente: entregar conocimientos desde una visión interdisciplinar sobre un ámbito cada vez más amplio y complejo de temas; modificar actitudes y comportamientos e incidir en la relación profesional de la salud-paciente y en el cambio de los modelos de asistencia en salud, y trasmitir los valores éticos más apropiados y necesarios para los profesionales de la salud y para la sociedad en general. También se aborda qué valores comunicar a los alumnos y cómo realizarlo, así como el modo de evaluar en esta disciplina.
\end{abstract}

Palabras clave: bioética, educación en valores, educación médica

\section{TEACHING BIOETHICS: HOW TO TRANSMIT KNOWLEDGE, ATTITUDES AND VALUES}

\begin{abstract}
This work presents teaching experiences and reflections about how to teach bioethics in a manner that will fulfill three goals, and, at the same time, the challenges for the teacher: to offer knowledge from an interdisciplinary view about a scope of subjects becoming every day wider and more complex; to modify attitudes and behaviours and to influence on the health-patient professional relationship and on the models' change in health care. The third goal consists on how to transmit the most appropriate ethic values for the health care professionals and for society in general. It also undertakes which values to communicate to students and how to carry it out, as well as how to evaluate in this discipline.
\end{abstract}

Key words: bioethics, values' teaching, medical education

\section{ENSINAR BIOÉTICA; COMO TRANSMITIR CONHECIMENTOS, ATITUDES E VALORES}

Resumo: Neste trabalho são apresentadas as experiências docentes e reflexões sobre como realizar o ensino da bioética de modo que cumpra com três objetivos, que são desafios desafios para o docente: transmitir conhecimentos a partir de uma visão interdisciplinar num âmbito cada vez mais amplo e complexo de temas; modificar atitudes e comportamentos e incidir na relação profissional da saúde-paciente e na mudança dos modelos de assistência em saúde. Além disso transmitir os valores éticos mais apropriados e necessários para os profissionais da saúde e para a sociedade em geral. Também se aborda que valores comunicar aos alunos e como fazê-lo, bem como o modo de avaliar esta disciplina.

Palavras chave: bioética, educaçấo em valores, educação médica.

\footnotetext{
Doctor en Filosofía. Profesor Adjunto del Centro de Bioética, Facultad de Medicina, Pontificia Universidad Católica de Chile, Chile Correspondencia: gibioetica@vtr.net
} 


\section{Introducción}

La bioética se ha incorporado de modo definitivo a los currículos de las diferentes carreras de ciencias de la salud en estos últimos años en Chile. Comenzó en medicina y enfermería y actualmente está en sus inicios en odontología ${ }^{1}$, kinesiología y en otras áreas afines, como trabajo social y terapia ocupacional ${ }^{2}$. Quizás es una asignatura aún pendiente en psicología, especialmente en todo el campo de la psicología clínica, pero se han dado ya algunos pasos ${ }^{3}$.

Es una disciplina reciente, con algo más de 30 años de evolución, pero cuenta ya con un desarrollo metodológico y didáctico importante. Quizás por su juventud ha estado muy abierta a las corrientes pedagógicas más actuales, aunque también puede deberse al reto que representa en sí misma, pues pretende algo nada sencillo: primero, entregar conocimientos desde una visión interdisciplinar sobre un ámbito cada vez más amplio y complejo de temas; segundo, cambiar actitudes y comportamientos e incidir en la relación profesional de la salud-paciente y en el cambio de los modelos de asistencia en salud. Finalmente, trasmitir los valores éticos más apropiados y necesarios para estos profesionales y para la sociedad en general.

Tanto la metodología de enseñanza como la actitud del profesor y la evaluación en las asignaturas de bioética tienen un contenido formativo al servicio de esa transmisión de valores, más que en otras disciplinas, y deben adecuarse a una estricta ética docente.

\section{El amplio campo de conocimientos interdisciplinares de la bioética}

No se trata de enseñar ética médica, ética en enfermería, deontología profesional, aspectos legales de estas disciplinas o, meramente, ética filosófica. Las propuestas

1 Se han realizado ya -promovidos por el Programa de Bioética de la OPS- tres jornadas de bioética y odontología, en Santiago (2005), Buenos Aires (2006) y Lima(2007). Otra iniciativa es la publicación del boletín Salud Oral y Bioética, del mismo Programa, del que se han editado dos números.

2 Como ejemplo, la reciente tesis de Licenciatura dirigida por la profesora Laura Rueda: Gaete C, Gallardo S, Ramírez C. Principios Bioéticos en la integración social de personas con discapacidad. Santiago de Chile: Escuela de Terapia Ocupacional, Universidad de Chile; 2006.

3 Además de la inclusión reciente en el currículo de algunas escuelas de psicología, se celebraron recientemente las III Jornadas Interdisciplinarias de Bioética, organizadas por el Centro de Bioética de la Facultad de Medicina y la Escuela de Psicología, de la Pontificia Universidad Católica de Chile. tienden a acercarse a algunos de esos extremos $(1)^{4}$, pero la bioética ha ido definiendo con bastante precisión su identidad (al menos conceptual, aunque el consenso no sea unánime o entendido por muchos) con una mirada más amplia e interdisciplinar y un estatuto epistemológico propio, por lo que se supone -y es exigible- que el profesor sea capaz de enseñar desde la perspectiva clínica y jurídica, desde la fundamentación filosófica de la ética y con la metodología y ámbito propio de la bioética. Además, debe hacerlo de un modo adecuado a las circunstancias culturales y sociales de su propio ámbito, en nuestro caso, de Latinoamérica, bien diferentes de otros $(2,3)$.

El campo de la bioética se ha ido ampliando cada vez más desde la ética clínica a las cuestiones de ética global y ambiental, y a los problemas de la ética de las instituciones y sistemas de salud. El mayor desarrollo en la docencia y en libros y artículos se ha producido en bioética clínica, pero las propuestas de una bioética global, centrada en la supervivencia en un mundo pluralista y complejo, merecen una reflexión juiciosa, pensando en la enseñanza de la misma(4). Por lo tanto, existe un amplio campo de contenidos, tanto por la materia en sí misma como por su abordaje desde muy diferentes disciplinas.

Ha habido bastantes propuestas respecto de los contenidos que debe abarcar. Francesc Abel (5) propone que "los objetivos docentes han de orientarse en torno a los tres ejes siguientes:

1) Desarrollo progresivo de la competencia profesional.

2) Armonización entre los valores del conocimiento técnico científico especializado y los valores del conocimiento global y humanístico de la persona.

3) La sinergia necesaria entre los objetivos de la política sanitaria y la forma como se instrumentan los medios o recursos para llevarla a término".

Añadiríamos que, hoy en día, la formación en bioética forma parte de la necesaria competencia del profesional de la salud, y que un objetivo importante es proporcionar elementos de juicio a los futuros profesionales para comprender los debates en bioética que se producen en su sociedad.

4 Salomone y Domínguez se centran en la dimensión singular y el campo normativo de la ética profesional, especialmente de los códigos de ética, con la propuesta de una ética de la responsabilidad como base de la deontología de las profesiones clínicas. 
El equipo de trabajo sobre la enseñanza de la bioética de la Asociación de Bioética Fundamental y Clínica, en España, propone seis módulos básicos: historia, fundamentación, metodología, relaciones asistenciales, principio y final de la vida, e investigación. Cada módulo puede impartirse en tres grados de nivel de conocimientos: introductorio, medio y de profundización(6). Este es el esquema que, en mayor o menor medida, se sigue en la mayoría de los programas de posgrado o magíster, o en los numerosos manuales de bioética existentes. Lo que varía es la proporción de contenidos dedicados a una u otra materia. En general, en Chile y en otros países se tiende a ampliar la parte de fundamentación y metodología, en detrimento de la bioética clínica. Quizás por la distribución de la docencia entre facultades o por otros motivos académicos o de concepción de la bioética, se amplían los contenidos teóricos. Sin embargo, debe advertirse el objetivo práctico de esta disciplina (una muy especial ética aplicada) así como la necesidad de formación de los profesionales para la actuación en el ámbito clínico y en los comités de ética.

Diego Gracia propone tres pasos en la formación de pregrado en medicina $(7,8)$. La ética básica, al comienzo, que "debe estudiar con una cierta detención y profundidad el tema de los juicios morales y el modo como pueden fundamentarse", con la articulación entre 'juicios' y 'principios' en un camino de ida y vuelta: "desde los juicios a los principios da lugar al problema de la 'fundamentación'; y el camino contrario, de los principios a los juicios, al problema de la 'metodología”. En el período clínico, la ética médica debe ser naturalmente clínica o aplicada, y también la ética profesional o deontología médica debe estar presente antes de terminar la formación profesional.

Esto exige al docente una permanente actualización. Todos hemos sido formados en una disciplina científica, muchas veces de manera bastante cerrada a otras, y debemos hacer el esfuerzo de conseguir una visión multidisciplinar. No se trata de que el médico ahora sea filósofo o de que el jurista se trasforme en médico, pero sí que éste incorpore los fundamentos de la ética filosófica y los fundamentos de la ciencia jurídica. Por otra parte, el filósofo debe entender la realidad clínica y los aspectos científicos de la investigación médica avanzada para aportar -en conjunto- una visión más amplia (y más completa) de esa realidad.
Este es el reto fundamental del docente de bioética a la hora de trasmitir contenidos. No puede reducirse sólo a trasmitir actitudes, a enseñar a deliberar, por importante que sea esto en su metodología, especialmente para entender y sacar experiencias de los casos ético-clínicos y en la formación de los estudiantes. Debe trasmitir, sobre todo en pregrado, conocimientos sobre ética, deontología profesional y legislación, y sobre ética clínica y ética global.

Es mucho el desarrollo conceptual producido ya en el ámbito académico en Latinoamérica, adecuado a nuestras sociedades. Los libros y artículos editados en bioética(9) y el desarrollo de la propia investigación exigen la puesta al día del docente en los conocimientos y publicaciones.

En el posgrado tiene un lugar prioritario el desarrollo de la capacidad de diálogo y deliberación morales(10-12) y el aprendizaje de metodologías de análisis de los dilemas ético-clínicos o para la toma de decisiones ético-clínicas(13). Pero también existe un contenido específico que trasmitir. Los alumnos deben ser capaces de realizar un análisis pormenorizado de los problemas éticos más frecuentes en sus distintas especialidades médicas o profesionales. Hoy, por ejemplo, existen libros específicos de bioética para la mayoría de las especialidades médicas reconocidas oficialmente y la etapa adecuada para su transmisión sería la de formación del médico especialista, o la especializada de posgrado en bioética. En la medida en que la educación en bioética se extienda más en el pregrado, tendrán que ser más profundos y especializados los contenidos que se impartan en los posgrados, también con la investigación realizada en las tesis de magíster y en las -aún relativamente escasas- tesis de doctorado sobre temas de bioética.

\section{Actitudes y comportamientos en la formación en bioética}

En necesario cambiar actitudes y comportamientos e incidir en la relación profesional de la salud-paciente y en los modelos de asistencia en salud. Cambiar actitudes en los estudiantes de medicina o en los profesionales es bastante más complejo.

\section{Conocer a los alumnos}

Por una parte, hay que intentar que superen algunas actitudes negativas con las que ingresan y que se mantienen también en bastantes profesionales: 
- Mentalidad no sólo científica sino cientificista: la ciencia lo explicaría todo y se daría a sí misma las reglas; nadie debe imponérselas y menos desde fuera de la propia ciencia.

- Intereses económicos, que a veces pueden sobreponerse al sentido de ejercicio de una profesión basada en el servicio a los demás. Esto no es lo propio de los estudiantes que ingresan, más idealistas, pero sí suele estar presente - por las responsabilidades que sienten más cercanas- en los que están terminando su formación o comienzan las especialidades.

- Actitud negativa ante la filosofía y la ética clínica: ¿para qué dedicar tiempo a la ética, sobre todo cuando se sienten tan exigidos en las asignaturas clínicas "duras" y no tienen, en ocasiones, un buen recuerdo de la filosofía que estudiaron en la educación media? Esto, por supuesto, no es igual entre estudiantes de posgrados en bioética, en los cuales existe generalmente el deseo de formarse en estas materias "filosóficas", precisamente por las limitaciones que experimentan en sus propias disciplinas.

- Pasividad en general en la formación académica: les "enseñan" anatomía, fisiología, patología, por ejemplo, y esperan que también se les "enseñe" bioética.

Pero también los alumnos traen incorporadas actitudes positivas sobre las que comenzar el proceso educativo:

- Cada vez más descubren el sentido social de su propia profesión, con una preocupación auténtica por la situación de desigualdad de la atención de salud en Chile y las enormes diferencias entre la medicina privada y la pública.

- Tienen una actitud crítica ante la comercialización de la medicina actual.

- Tienen curiosidad intelectual y una actitud abierta al aprendizaje. Algunos desean recuperar antiguos intereses humanistas que dejaron olvidados años atrás. Además, sienten que la bioética es algo novedoso.

- Los alumnos de primer año, especialmente, ven la asignatura de Bioética o de Fundamentos Filosóficos de la Medicina como las únicas que se acercan a la praxis médica directa en un currículo lleno de materias básicas. Un poco más tarde sienten la falta de preparación ante los dilemas éticos que empiezan a encontrar de golpe en las prácticas.

\section{Promover las actitudes y la capacidad de diálogo}

Sobre todo, las actitudes imprescindibles en todo diálogo bioético: "respeto al otro, tolerancia, fidelidad a los propios valores, escucha atenta, actitud interna de humildad; reconocimiento de que nadie puede atribuirse el derecho a monopolizar la verdad y que todos hemos de hacer un esfuerzo para ser receptivos, esto es, aceptar la posibilidad de cuestionar las propias convicciones desde otras posiciones y lo razonable de otros argumentos. En otras palabras, se necesita la escucha recíproca, el enriquecimiento de la competencia profesional interdisciplinar y la autenticidad de los acuerdos. Los dogmatismos científicos o espiritualistas y las visiones reduccionistas son un auténtico estorbo. La ignorancia convierte en imposible el diálogo bioético"(5).

Para promover la educación moral en general, y la bioética en particular, la discusión de dilemas morales puede ser un buen recurso metodológico. En una época en la que urge desarrollar la capacidad dialógica de las personas para resolver los problemas sin hacer uso de la imposición o la violencia, es muy útil conocer este procedimiento educativo, válido para suscitar el diálogo y para adoptar posturas razonadas y razonables en el terreno de los valores, las actitudes y la conducta(14).

\section{Acciones educativas para el cambio de actitudes}

Señalaré sólo algunas dictadas por la experiencia más reciente:

- Adecuar la metodología a los avances en pedagogía de la ética desde las teorías del desenvolvimiento moral de Piaget y Kohlberg, entre otras.

- Interesarnos por los valores que realmente viven los estudiantes de medicina para comprender mejor las posibilidades y los límites de su educación $\operatorname{moral}(15,16)$.

- Participar en su aprendizaje ético-clínico, estar con ellos en sus prácticas, a través de tutorías con grupos pequeños; fundamentar el aprendizaje en casos que descubran no sólo métodos de resolución y decisión, sino actitudes en los pacientes y en los equipos de salud; que relacionen lo que enseñamos con lo que suele pasar en sus prácticas.

- Facilitar que puedan narrar sus "casos". Descubriremos con asombro los numerosos dilemas éticos 
a los que se ven enfrentados, tanto por la situación de los pacientes como de los profesionales que les atienden como docentes clínicos. Tenemos que conocer mejor las situaciones por las que atraviesan para formarles mejor.

- Usar el método del aprendizaje basado en problemas para el cambio de actitudes. Unir el aprendizaje clínico con el aprendizaje ético en tutorías de grupos pequeños, en las cuales se complementa la resolución clínica del caso con la resolución de los problemas ético-clínicos que van apareciendo(17).

- Desarrollar tutorías con casos ético-clínicos y que el alumno exponga un caso que le haya sucedido o del que tenga conocimiento. De esta forma integra la ética con la praxis clínica que esté aprendiendo.

- Utilizar medios audiovisuales diversos que acerquen a los alumnos a los problemas reales de la sociedad. Hay muy buena experiencia con cine(18): "El buen cine es el arte de contar historias de gente posible. Más allá de su dimensión de entretenimiento, es un espejo donde la gente se busca y quiere mirarse, y a través del cual ve también el mundo que le rodea”. Es un instrumento para la enseńanza que pretende acercarse a los interrogantes y la vida de los estudiantes. Usar también comentarios de noticias o informes de prensa, casos reales que hayan sido analizados por los comités de ética, con la debida confidencialidad, material didáctico específico y claro, entre otros recursos(19). Las clases magistrales pueden ayudar a trasmitir contenidos, pero cuando se trata de cambiar actitudes y trasmitir valores resultan bastante menos eficaces que el trabajo en grupos pequeńos interactuando con el profesor.

- En la etapa de residencia es especialmente importante que los estudiantes puedan dialogar con los tutores sobre los aspectos éticos de la clínica. Los alumnos de $4^{\circ}$ y $5^{\circ}$ año de medicina tienen todavía la óptica del paciente más que la del médico ante los problemas éticos. Los residentes ya no, porque muchas veces ayudan y a veces "sustituyen" a los médicos en la resolución de muchos dilemas éticos de información, consentimiento informado, educación, entre otros.

- Formar también a los formadores, a los docentes clínicos. En nuestra experiencia en la Facultad de Medicina de la Pontificia Universidad Católica de Chile han sido muy eficaces los cursos-taller de Ética Clínica para docentes clínicos. Más de la mitad de los médicos que ejercen como profesores o tutores en la facultad, y en los hospitales donde los alumnos realizan las prácticas, han cursado ya este taller. Nuestros estudiantes sólo cambiarán sus actitudes si también ven cambios en sus docentes clínicos. Damos por supuesta la importancia de las propias actitudes del docente con sus alumnos y la coherencia entre lo que trata de enseñar y lo que practica en la relación profesor-alumno(20).

\section{Cómo trasmitimos valores en bioética}

\section{Conseguir que quieran "bacer"}

Trasmitir y formar en valores. Este es el reto más importante para un educador y para un profesor de bioética. Llegar a lo íntimo de la formación moral del estudiante y ayudarle a incorporar, mejorar o jerarquizar de un mejor modo los valores éticos que ya tiene y los que va a ir adquiriendo a lo largo de su carrera. Permitirle "entrar" en esa "disposición moral de ánimo" que Max Scheler define como la actitud o motivo que nos lleva a querer hacer algo, antes incluso de tener la intención de hacerlo, y que sería previa a la acción moral y a la explicitación de valores morales(21).

No es posible cambiar directamente esa disposición moral esencial del ánimo en el estudiante, pues sólo conseguiríamos, quizás, una conducta hipócrita, realizada de cara a sus profesores, a sus compañeros o a los pacientes. No porque "expliquemos" muy bien cuáles son los valores éticos, el alumno los va a incorporar de modo racional. Se necesita una acción indirecta, de modo que sea el propio alumno quien vaya "descubriendo" esos valores, los matices que presentan en la acción clínica, las incidencias de cada uno de ellos en la propia actuación personal.

No sirven los mandatos u órdenes, ni siquiera la mera demostración de la racionalidad de lo que proponemos. Es necesario proporcionar indicaciones educativas que sugieran acciones para que el estudiante las lleve a cabo libremente y, de ese modo, se perfeccione como persona individual. El educador puede proponer no acciones sino intenciones o campos de acción, no algo que el estudiante "debe hacer", sino que debe "querer-hacer". Lo importante de la acción educativa -al menos en bioética- no es conseguir que el estudiante haga algo, sino que quiera por sí mismo hacer algo, porque lo encuentra valioso como futuro médico y como persona. 


\section{La importancia de los modelos}

Algo esencial para la formación moral son los modelos. El "seguimiento" de modelos es el medio más común y adecuado en la nińez y la adolescencia, y sigue siendo válido después. Esto convierte la tarea educativa en algo mucho más exigente para todos los que colaboran en la formación profesional de los estudiantes de medicina. Nuestra forma de actuar es la mejor "clase" que podemos dar a los alumnos.

Pero no basta sólo con que se propongan seguir determinados modelos. Es fundamental el respeto por la autonomía del alumno. Es clave que el propio alumno participe de su formación como médico, sin ninguna pasividad, cuestionándose de modo autónomo sus propios comportamientos y valores.

Si en un grupo de tutorías de ética clínica, alguno de los alumnos no participa en el diálogo ético e, implícitamente, rechaza al profesor y a sus compañeros como "interlocutores válidos", probablemente es porque piensa que éstos no tienen nada que decirle, porque él ya tiene sus valores bien definidos y "no quiere" cambiarlos o cuestionarlos. Como profesores debemos conseguir que todos participen de ese diálogo racional autónomo, proceso imprescindible para conseguir una personalidad moral adulta(22).

Y aquí pueden ayudarnos mucho esas características metodológicas de la propia bioética: el diálogo racional como medio de avanzar en los planteamientos éticos(23), los diferentes métodos de deliberación para la toma de decisiones ético-clínicas y la resolución de casos que ayudan a profundizar también en la fundamentación de las propias convicciones y valores morales.

\section{Entonces, ¿qué valores?}

Ya hemos hablado de algunos valores que podemos llamar "instrumentales", como la capacidad de diálogo, la participación activa, la capacidad de tomar decisiones autónomas, entre otros. Pero éstos no bastan. Debemos promover otros, para no "estar rodeados de científicos, intelectuales y profesionales, sobresalientes en ciencias y tecnologías pero deficientes en valores morales, culturales y sociales" (24). La universidad no puede renunciar a su función educadora, con el máximo respeto a la autonomía de los alumnos y profesores. Debe promover valores, pues "sólo desde la educación es posible ejercer la libertad, o -en palabras de Séneca- 'la única libertad es la sabiduría"'(25).

La formación de la inteligencia, de la voluntad y de los valores éticos es aún más necesaria en nuestros días, en especial en el ámbito de la bioética. Es posible educar en valores(26). Algunos son comunes a todos en una sociedad democrática, pero otros tienen que ver más directamente con los profesionales que intervienen activamente en el cuidado de la vida y la salud. Consideramos más fundamentales los que están en la base de los principios bioéticos: el respeto a la vida y la salud como bienes básicos es un valor que se realiza a través de la no maleficencia y la beneficencia; el respeto a los derechos de otros -y en especial de los más vulnerables-es uno de los valores fundantes del principio de justicia; la madurez moral y la responsabilidad por las consecuencias de las propias acciones van muy unidas al ejercicio de la libertad, en el principio de autonomía; poner a la persona del otro por encima de los legítimos intereses propios, tender a la excelencia en los cuidados de salud, la compasión y el cuidado de la vida frágil, y la solidaridad individual y social fundamentan, finalmente, el principio de beneficencia.

\section{La evaluación en la docencia de bioética}

Es posible medir el aprendizaje de los contenidos trasmitidos, pero es más difícil evaluar la adquisición de las actitudes y comportamientos que forman parte de los objetivos docentes, y más aún la incorporación o profundización en los valores.

Se han desarrollado y aplicado a la enseñanza de la ética médica técnicas que ya se utilizaban en otros ámbitos de la docencia médica, como los exámenes OSCE(27) para medir los comportamientos éticos, aunque cabe la posibilidad de que el alumno realice estos comportamientos para la finalidad evaluativa y no porque efectivamente los haya incorporado reflexivamente.

Se han realizado algunas investigaciones con metodología cualitativa para acercarse a conocer la profundidad del impacto de la enseñanza de la bioética y los cambios en los comportamientos de los alumnos de medicina(28) o en médicos residentes de primer año(29). Quizás uno de los medios más eficaces sean las propias autoevaluciones de los alumnos, ya como objeto de investigación cualitativa -como en un reciente estudio con internos de primer año en la Universidad Diego Portales(17) - o incluidas en la propia metodología de 
los cursos de ética, con autoseguimiento sistemático del desempeño estudiantil en cada sesión de clase en el aula, como en un reciente trabajo en la Universidad de Caldas(30). Éste corresponde a un trabajo participativo, activo y reflexivo sobre el propio comportamiento de los alumnos, valorado a la luz de criterios establecidos en la normatividad institucional, en la Declaración de Derechos Humanos, en los Derechos Constitucionales y en principios pedagógicos y éticos.

\section{Referencias}

1. Salomone G, Domínguez ME. La transmisión de la ética: clinica y deontología. Volumen I. Fundamentos. Buenos Aires: Letra Viva; 2006: 173.

2. Garrafa V, Kottow M, Saada A, (coords.) El estatuto epistemológico de la bioética. Un enfoque latinoamericano. México: UNESCO e Instituto de Investigaciones Jurídicas de la UNAM; 2005.

3. Llano A. (editor) ¿Qué es Bioética? Según notables bioeticistas. Bogotá: 3R Editores; 2001.

4. Escobar J. (coord.) Elementos para la enseñanza de la Bioética. Bogotá: Universidad El Bosque; 1998.

5. Abel F. Bioética: diálogo interdisciplinar. Cuadernos de Bioética 1999; 37(1): 11-16.

6. Asociación de Bioética Fundamental y Clínica. La educación en Bioética de los profesionales sanitarios. Madrid: Ed. Lormo; 1999.

7. Gracia D. Fundamentación y enseñanza de la bioética. Bogotá: Editorial El Búho; 1998: 175-183.

8. Cely G. Bioética y Universidad. Bogotá: Centro Editorial Javeriano CEJA; 1997.

9. León FJ. Bibliografía sobre Bioética en los últimos años. 500 recensiones de libros editados en español. $2^{\mathrm{a}}$ ed. electrónica. Madrid: Asociación Española de Bioética; 2007.

10. Gracia D. La deliberación moral, el método de la ética clínica. Medicina Clínica 2001; 117 (1): 18-23.

11. Maliandi R. Ética discursiva y ética aplicada. Reflexiones sobre la formación de profesionales. Revista Iberoamericana de Educación 2002; 29: 105-130.

12. Bellver V. Por una bioética razonable. Granada: Comares; 2006.

13. Gracia D. Procedimientos de decisión en ética clinica. Madrid: EUDEMA; 1991.

14. Cantillo J, Domínguez A, Encinas S, et al. Dilemas Morales. Un aprendizaje de valores mediante el diálogo. Valencia: Nau Llibres; 2005.

15. Rego S. A Formaçâo Ética dos Médicos. Saindo da adolescencia com a vida (dos outros) nas mâos. Río de Janeiro: Fiocruz; 2005.

16. Gómez AI, Maldonado CE. (comps.) Bioética y educación. Investigación, problemas y propuestas. Bogotá: Universidad del Rosario; 2005.

17. Mesina M, León F, Lillo R. Formación en Bioética de estudiantes de Medicina: experiencias del Aprendizaje Basado en Problemas en la Universidad Diego Portales. Santiago de Chile: Comunicación en IV Congreso de Educación Médica; 2007.

18. Tomás MC, Tomás GM. La vida humana a través del cine. Cuestiones de Antropología y Bioética. Madrid: Eiunsa; 2004.

19. Calva R. Bioética. México: McGraw-Hill; 2006.

20. Aluja M, Birke A, (coord.) El papel de la ética en la investigación cientifica y la educación superior. 2a ed. México: Academia Mexicana de Ciencias, Fondo de Cultura Económica; 2004.

21. Sánchez-Migallón S. La persona humana y su formación en Max Scheler. Pamplona: Eunsa; 2006.

22. Cortina A. Ética minima. $4^{a}$ ed, Madrid: Tecnos; 1994: 207-214.

23. Lolas F. Bioética. Santiago de Chile: Editorial Universitaria; 1998.

24. Llano A. (editor) Bioética y educación para el siglo XXI. Bogotá: Pontificia Universidad Javeriana; 2006.

25. Melendo T, Millán-Puelles L. La pasión por la verdad. Hacia una educación liberadora. Navarra: EUNSA; 1997.

26. Melich JC, Palou J, Poch C, Fons M. (eds.) Responder del otro. Reflexiones y experiencias para educar en valores éticos. Madrid: Editorial Síntesis; 2001.

27. Florenzano A, Mosso L, Pérez P. Examen clínico objetivado estructurado en evaluación de ética clínica: ¿es posible? Santiago de Chile: Comunicación en IV Congreso de Educación Médica; 2007. 
Enseñar bioética: cómo trasmitir conocimientos, actitudes y valores - Francisco Javier León Correa

28. Salas S, (investigador responsable) Análisis del impacto de la enseñanza de la bioética en las actitudes ético clínicas de alumnos de Medicina. Santiago de Chile: Vicerrectoría Académica, Pontificia Universidad Católica de Chile; 1998.

29. Sogi C, Zavala S, Ortiz P. ¿Se puede medir el aprendizaje de la ética médica? Anales de la Facultad de Medicina, Universidad Nacional Mayor de San Marcos 2005; 66 (2): 174-185.

30. Franco ZR. La evaluación ética en la educación para el desarrollo humano. Caldas: Editorial Universidad de Caldas; 2006.

Recibido: 25 de marzo de 2008

Aceptado: 6 de abril de 2008 